\title{
Burden, Quality of Life, and Palliative Care for Family Caregivers of Individuals with Advanced Liver Disease: a Systematic Literature Review
}

\author{
Christopher D. Woodrell ${ }^{1,2}$ (D) Arnab Mitra ${ }^{3} \cdot$ Andrew Hamilton $^{4} \cdot$ Lissi Hansen $^{5,6}$
}

Accepted: 9 May 2021 / Published online: 12 November 2021

This is a U.S. government work and not under copyright protection in the U.S.; foreign copyright protection may apply 2021

\begin{abstract}
Purpose of Review Identify and describe prior studies of family caregiver quality of life, health, and burden experienced while caring for patients with advanced liver disease and the possible role of palliative care.

Recent Findings Thirty-six articles were identified that met inclusion criteria. Studies identified included cross-sectional, longitudinal, and prospective intervention studies, with a high proportion of qualitative and descriptive research. Resulting studies addressed type of family caregiver burden, including physical, psychological, and financial causes of burden, including HE, lack of information and communication, and unpredictability of the disease course. Few prospective studies were identified. The interventions described included nurse-led palliative care, mindfulness-based stress reduction, and coping interventions.

Summary Little has been published on the nature of family caregiving, burden, quality of life, or the role of palliative care for family caregivers of people with advanced liver disease. Prospective studies that address unmet family caregiver needs, including palliative care interventions, are lacking.
\end{abstract}

Keywords End-stage liver disease $\cdot$ Caregiver burden · Quality of life $\cdot$ Palliative care $\cdot$ Liver transplant $\cdot$ Hepatocellular carcinoma

\section{Introduction}

End-stage liver disease (ESLD) arises generally in the setting of cirrhosis, defined as irreversible scarring of the liver

This article is part of the Topical Collection on Palliative Care

Christopher D. Woodrell

christopher.woodrell@mssm.edu

1 Brookdale Department of Geriatrics and Palliative Medicine, Icahn School of Medicine at Mount Sinai, 1 Gustav L. Levy Place, Box 1070, New York, NY 10029, USA

2 Geriatric Research, Education and Clinical Center, James J. Peters Veterans Affairs Medical Center, Bronx, NY, USA

3 Division of Gastroenterology \& Hepatology, Oregon Health \& Science University, Portland, OR, USA

4 Library, Oregon Health \& Science University, Portland, OR, USA

5 School of Nursing, Oregon Health \& Science University, Portland, OR, USA

6 Veterans Affairs Portland Healthcare System, Portland, OR, USA resulting from chronic inflammation and/or injury. Cirrhosis exists in two states: compensated and decompensated [1]. Signs of decompensation include ascites, hepatic encephalopathy (HE), bleeding related to portal hypertension, and kidney failure. Hepatocellular carcinoma (HCC), the most common type of primary liver cancer, is an additional complication of ESLD. Approximately 4.5 million adults in the USA were diagnosed with liver disease in 2018, with 41,473 deaths in 2017 [1]. (CDC, National Health Interview Survey, 2018). Mortality risk increases depending on number and severity of decompensating events $[1,2]$. Liver transplant can be curative for some people facing decompensated cirrhosis and/or HCC. However, many patients are not able to receive a transplant due to medical and/or psychosocial factors [3]. Advanced liver disease results in physical and emotional stressors for patients and their families because of 
the physical manifestations of liver disease and uncertainty associated with its course.

Informal caregiving, often provided by an unpaid family member, includes assistance with activities of daily living or instrumental activities of daily living. We refer to those who provide this care as family caregivers; however, some are not legally family members. In the USA, it is anticipated that approximately 27 million people will need care by 2050 [4]. The immense burden of liver disease and associated physiologic, psychological, and social complexities pose threats to the quality-of-life (QOL) experienced by both patients and family caregivers [5]. Furthermore, data have increasingly demonstrated interdependence of

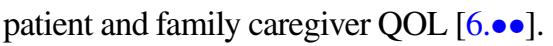

Palliative care is specialized care that focuses on quality of life and can be delivered alongside disease-directed therapies, and is delivered by a team of healthcare professionals, including chaplains, nurses, physicians, and social workers [7]. A previous systematic review of patient, family, and health professionals' experience and the role of palliative care in the context of cirrhosis encompassed over 1,413 patients but only 31 family caregivers in aggregate, suggesting a significant knowledge gap in the family caregiver experience and the role of palliative care [8.•].

This systematic review focuses exclusively on prior studies of family caregiver QOL, health, and burden experienced in caring for patients with advanced liver disease, and the possible role of palliative care to improve family caregiver outcomes. We included studies of family caregivers of patients who are awaiting, have undergone, or are not eligible for liver transplant, including those with or without HCC. Because of heterogeneity of these conditions in the search, we refer broadly to "advanced liver disease" to include these conditions. We propose a conceptual model, identify possible gaps, and suggest future directions.

\section{Methods}

\section{Study Design}

Based on the Preferred Reporting Items for Systematic Reviews and Meta-Analyses (PRISMA) guidelines, we conducted a systematic review of literature related to the role and experience of informal family caregivers for people with advanced liver disease and the role of palliative care [9].

\section{Search Strategy}

Our search was conducted by an experienced health science education and research librarian (AH) using a comprehensive search string within CINAHL, MEDLINE, EMBASE, and PsycINFO. Verbatim search codes are included in the supplementary materials (Table $\mathrm{S} 1$ ). References published on or before July 14, 2020 are included. References were uploaded to RAYYAN, a web-based systematic review application [10].

\section{Selection of Studies}

Abstracts of the resulting publications were reviewed in RAYYAN independently by each of three investigators (CDW, AM, LH). Articles where consensus was not reached initially were discussed via video teleconference until consensus was reached. Inclusion criteria were (i) original scientific articles, (ii) in English, and (iii) inclusion of data related to experiences and outcomes of family caregivers for people with advanced liver disease. We included systematic reviews, case series, patient-focused studies if data at the level of the family caregiver were included, observational and prospective studies, and qualitative, quantitative, and mixed-methods studies. Publications were included regardless of the country of origin. Exclusion criteria were (i) scientific abstracts or oral meeting presentations, (ii) narrative reviews, (iii) editorials, (iv) case reports, (v) not published in English, (vi) did not report family caregiver data (e.g., family caregiver QOL or burden measures), (vii) focus on caregivers of patients with conditions other than advanced liver disease (e.g., other cancers or types of organ failure), and (viii) focus on caregiver outcomes postLT more than a year following LT.

At least two investigators reviewed the complete text of each article (CDW, AM, LH) for consistency with inclusion and exclusion criteria. Any difference in determination was resolved by article review over video teleconference. Given the limited number of articles on the topic of interest, no articles were excluded based on the year of publication. Due to the heterogeneity of outcomes and mostly lower levels of evidence (i.e., not randomized trials), we have not synthesized the data reported in our resultant articles. Furthermore, because of the heterogeneity of population, settings, methods, and outcomes, we have elected not to compare risk of bias across the studies in this review.

\section{Data Analysis}

Resulting articles were tabulated in Table 1 . Survey instruments used were reviewed and are in Table 2. The findings and salient themes were identified. Major themes were used to create a modified conceptual model of family caregiving for people with advanced liver disease (Figure 2).

\section{Results}

\section{Overview of Results}

A flowchart of search results, and article review and inclusion is shown in Figure 1. The initial search revealed 731 unique entries. Thirty-six studies (Table 1) met inclusion 


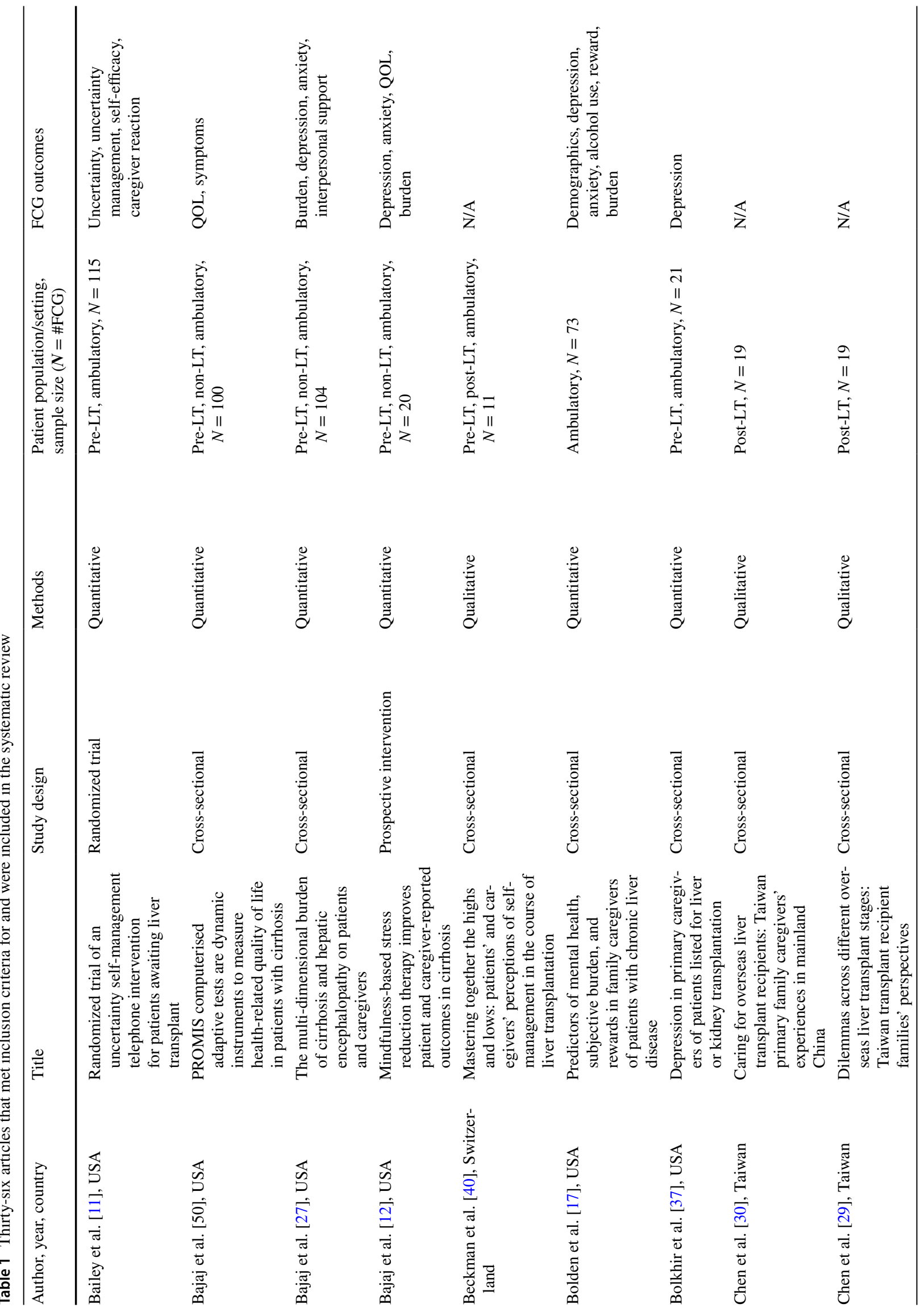




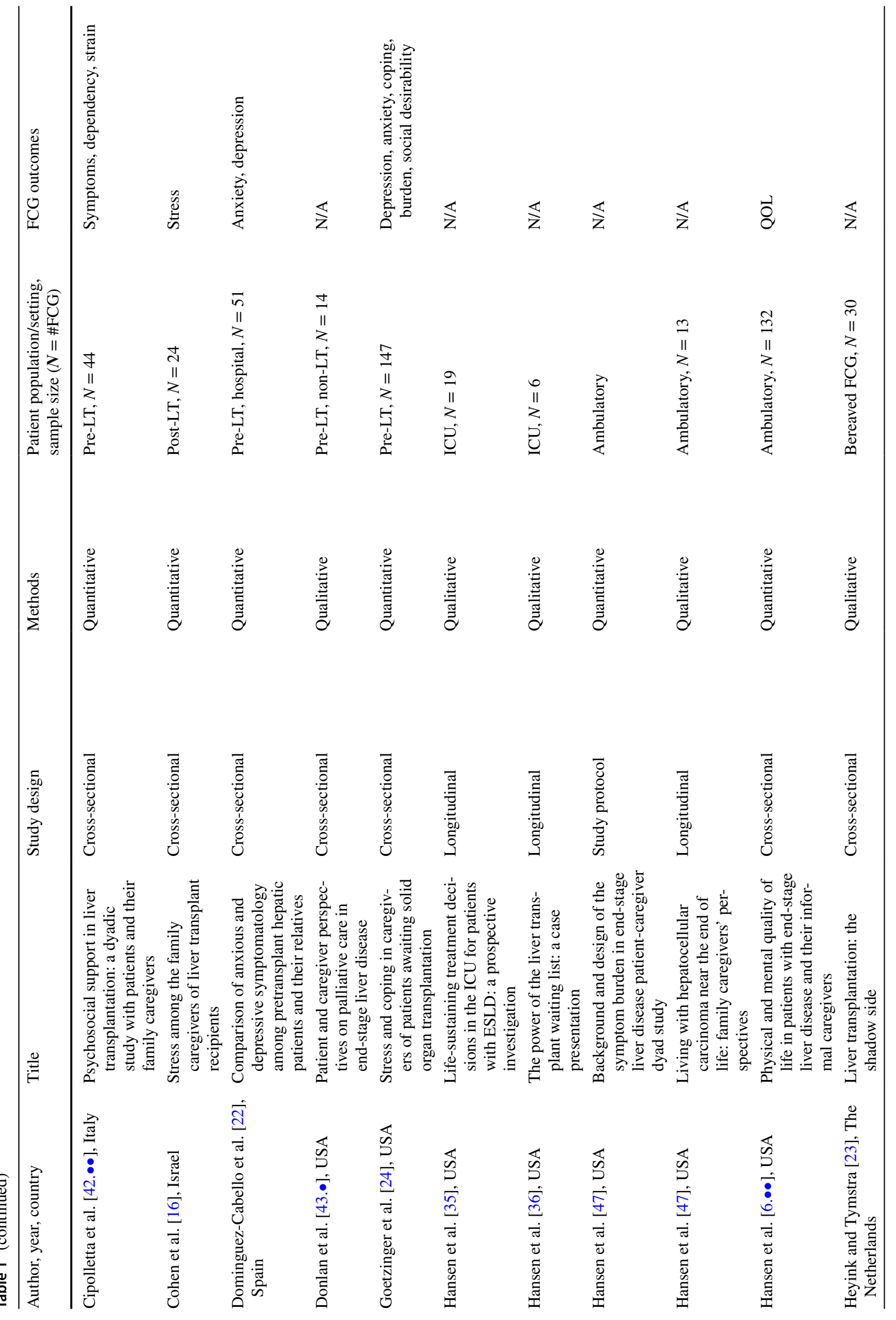




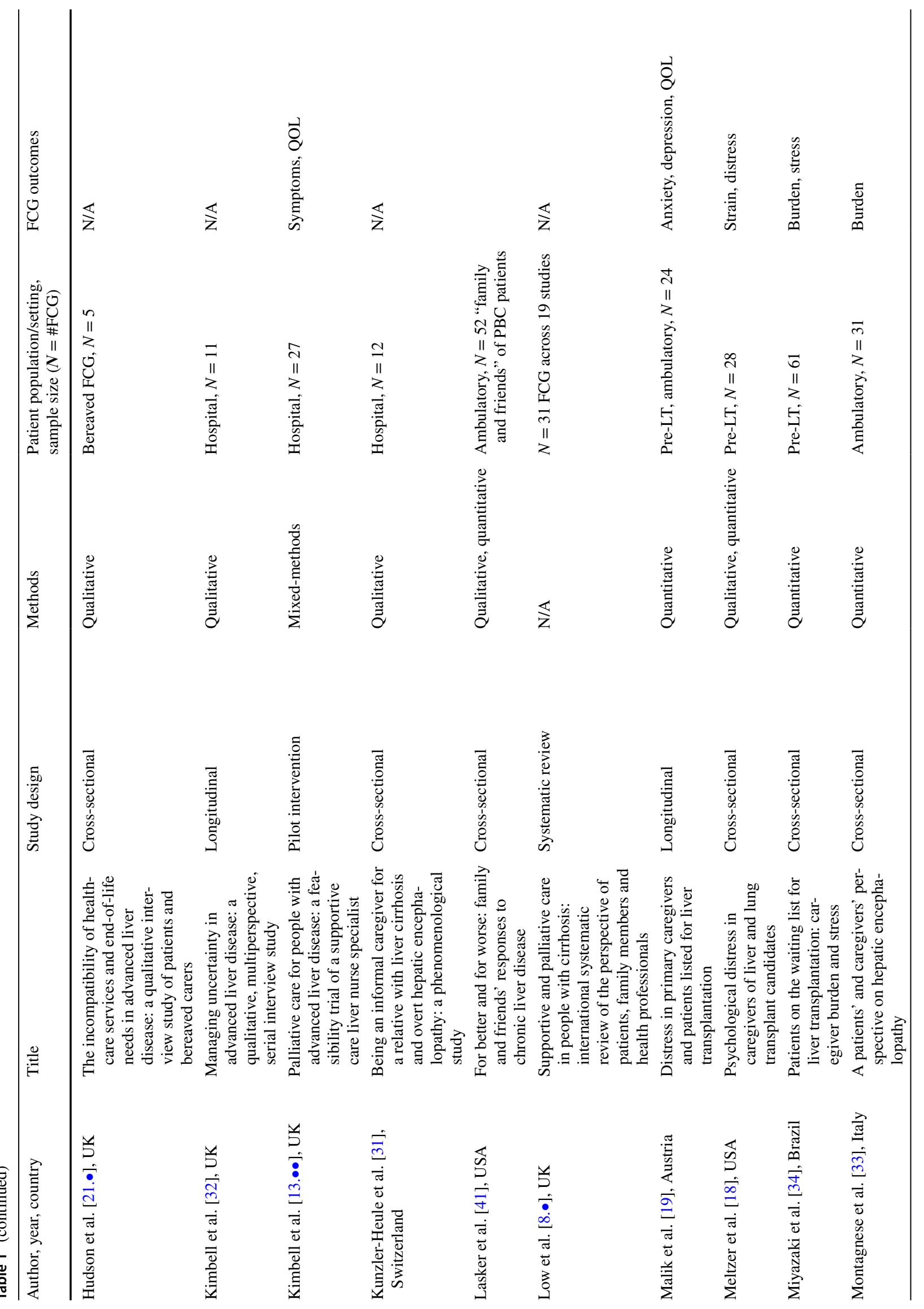




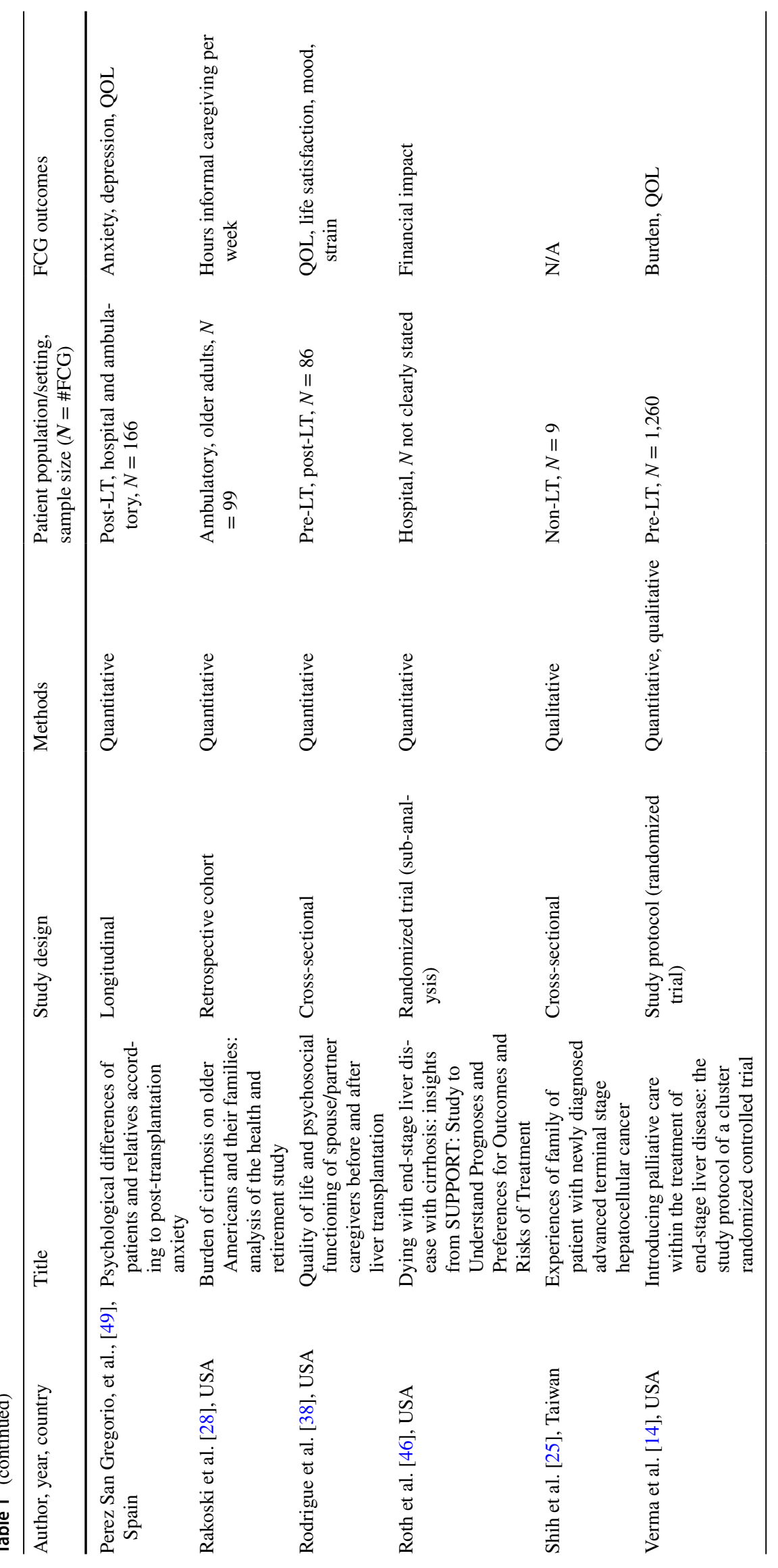




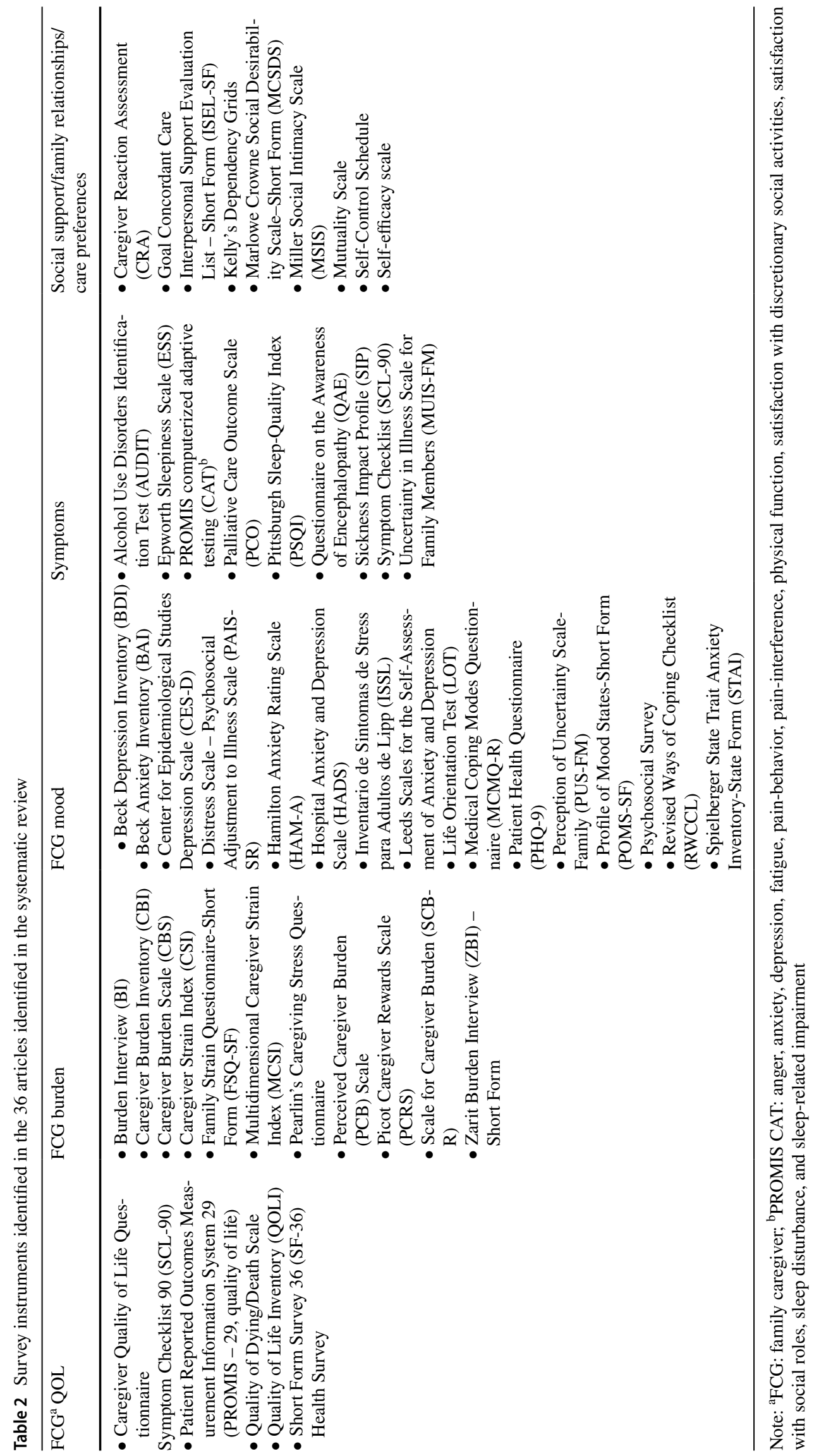


criteria and included the following types of studies: crosssectional (22), longitudinal (6), prospective interventions (4), study protocols (2), retrospective cohort (1), and systematic review (1). Methods employed were qualitative (12), quantitative (19), both qualitative and quantitative (3), and mixed-methods (in which the qualitative and quantitative data are formally triangulated or mixed) (1). Of completed published studies, family caregiver sample sizes ranged from six to 166 . Populations of family caregivers included those caring for patients with advanced liver disease, awaiting liver transplant, had had a liver transplant, and who had HCC. The majority of our resultant studies included a study population described as either pre-LT or unspecified. Of note, we included post-LT ( $N$ $=4$ ) studies that evaluated caregiver outcomes up to a year following transplant to include the illness trajectory around transplantation.

The majority of the quantitative studies were cross-sectional and employed diverse survey instruments (Table 2). Few of the tools had been validated in a sample of family caregivers exclusively caring for people with advanced liver disease. Our search revealed only three completed

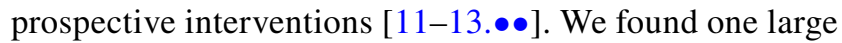
randomized trial protocol that is still underway [14].

In the studies reviewed, family caregivers are most frequently women and spouses $[15,16]$. Bolden and Wicks describe a population of family caregivers of people with liver disease: $78 \%$ women, $41 \%$ full-time employed, mean age of 48 years, 12 years average education level, and average of 6 years of care provided [17]. Overall, when examined alongside a normative sample of the general population, or family caregivers of people with Alzheimer's dementia, family caregivers of people with advanced liver disease reported lower QOL [18].

\section{Types of Family Caregiver Burden}

Burden featured prominently in our search results across the trajectory of advanced liver diseases, including before and after liver transplantation, near the end of life, and in the context of HCC $[19,20]$. The types of burden included (i) physical, (ii) psychological, and (iii) financial.
Fig. 1 Literature review flow diagram of a systematic review conducted using PRISMA guidelines9; articles were identified using multiple core biomedical databases as described in the "Methods" section, reviewed for inclusion and exclusion criteria, with 36 articles included (bottom-most box)
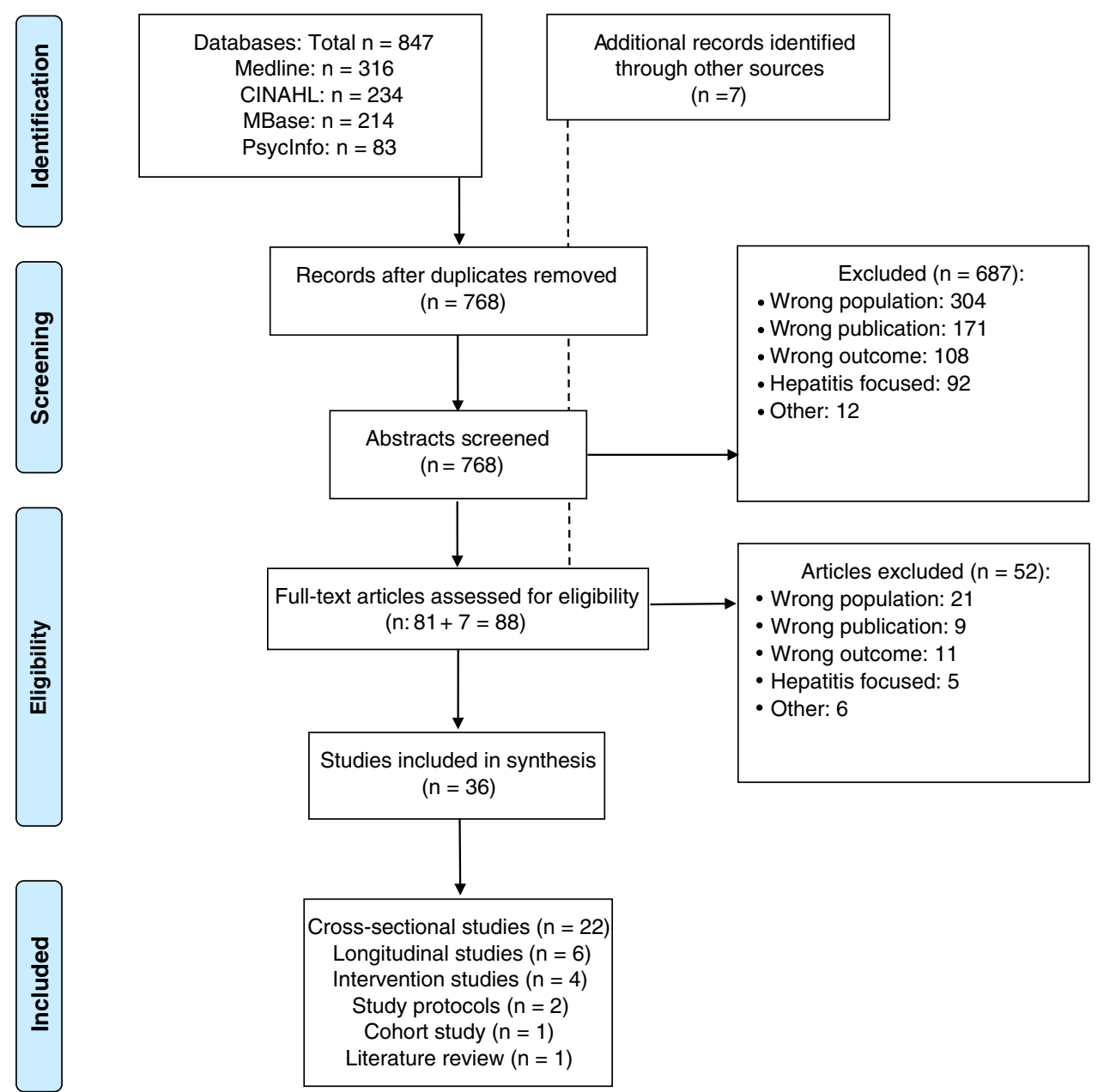


\section{Physical Burden}

Family caregivers describe de-prioritizing their own needs, included those that are physical. Ailments in the context of caregiving include insomnia, mechanical injuries from lifting, and neglect of their own healthcare needs and appointments [21.•].

\section{Psychological Burden}

Many caregivers experienced psychological symptoms, including depression and anxiety $[19,22]$. One study described experiences of family members $(n=9)$ of patients with newly diagnosed terminal $\mathrm{HCC}$ which centered around four themes: blaming oneself, disrupting the pace of life, searching all possible regimens, and not letting go [25].

\section{Financial Burden}

Family caregivers also experienced financial burden from caregiving responsibilities. Caregivers of patients with ESLD reported quitting their job, losing income, and depleting savings in the last 6 months of a patient's life [26]. HE was associated with less yearly family income, with MELD score significantly correlated with caregiver report of strain finances [27]. Health and Retirement Study data showed that older Medicare beneficiaries with cirrhosis required more weekly hours of caregiving than matched controls [28]. Financial hardship affected African American and women caregivers in particular [17]. Family caregivers reported stress associated with travel for specialized care, including upheaval of everyday life and financial effects $[29,30]$.

\section{Factors Leading to Increased Family Caregiver Burden}

We grouped causes of burden for family caregivers of people with advanced liver disease.

\section{Hepatic Encephalopathy}

Patient symptoms and behaviors, and in particular HE, caused feelings of being overwhelmed, worsening of physical health, and increasing burden among family caregivers $[21 . \bullet, 25,27,31,32]$. HE is a spectrum of neuropsychiatric symptoms including sleep and mood disturbances, confusion, somnolence, and in severe cases coma. Higher rates of burden, especially affecting mental health, have been documented in those caring for patient with HE tending to affect spouses more than other types of family caregivers [27]. These findings were corroborated in a study demonstrating positive correlation in caregiver burden and HE severity [33].

\section{Unpredictability and Uncertainty}

The unpredictability of the clinical course of ESLD also can lead to caregiver burden, especially in the setting of liver transplant consideration. Previous studies showed increased anxiety in family members of patients awaiting liver transplant [23]. Family members of transplant recipients expressed anxiety about the waiting process and the uncertainty of outcomes [29].

Several studies cited uncertainty as a source of family caregiver distress, including unpredictable prognosis. Kimbell and colleagues reported prognostic uncertainty as a dominant source of distress among family caregivers [32]. They cited unpredictability of physical deterioration, poor communication about prognosis, and complex care needs as contributors to distress. Family caregivers also reported feeling overwhelmed by poorly understood symptoms associated with advanced liver disease [31]. Family caregiver physical QOL has been shown to be negatively impacted by caring for older patients and the presence of refractory ascites, and feelings of uncertainty $[6 . \bullet \bullet]$.

Many patients with ESLD seek transplantation as a cure. However, not all candidates receive a liver, and the waiting process can lead to uncertainty and distress. In one study that included 28 family caregivers of liver transplant candidates, common stressors were waiting and uncertainty [18]. Uncertainty can also impact mental health: a longitudinal study of depression and anxiety among family caregivers awaiting liver transplantation showed increased rates of depression in the first 2 months of waiting, and rising rates of family caregiver anxiety over subsequent months [19]. Uncertainty also significantly affects the experience of family caregivers of people suffering from advanced HCC [20].

\section{Unpreparedness}

Family caregiver feelings of unpreparedness had a negative impact on the experience of family caregivers of people with advanced liver diseases. Family caregivers of liver transplant recipients reported feeling unprepared to address crisis situations, respond to changing patient mood, or manage medication and food preparation [34]. In a longitudinal qualitative study with family caregivers of HCC patients close to the end of life, participants reported that they felt unprepared, uncertain, and lacked information. They faced challenges around medication management, how to address crises, and lack of information about what to expect [20]. Family caregivers also report inadequate medical services and resources available near the end of patients' lives [21.•]. 


\section{Communication and Information}

Insufficient communication with healthcare providers has also been shown to cause distress. In one study, family members had limited disease understanding, wanted to know more, and communication between health professionals and patients/family members was difficult; stigmatization was cited as a barrier to communication [8.•]. In one study in the intensive care unit, families reported lack of inclusiveness in decision-making and information provided; in response, families developed protective information-gathering practices [35]. In a study of family caregiver experiences with "status 7" listing (suspension from the liver transplant waiting list), inconsistent communication led to emotional turmoil, frustration, confusion, and stress [36].

In a prospective, longitudinal study of caregivers of patients with end-stage HCC, caregivers wanted information about HCC and treatments, felt uncertain and unprepared for end of life care, and struggled with symptom interpretation [20]. Similarly, qualitative data demonstrated that the connection between observed symptoms (HE) and liver disease is not necessarily apparent to family caregivers [31]. The unpredictability of the onset of symptoms and physical deterioration and poor communication around these issues adversely affected family caregivers' experience [32].

Transmission of information may also affect the quality of a patient's death; when patients with advanced liver disease became critically ill, families reported different perceptions of how sick their loved one was compared to the medical team. Family caregivers often felt that prolonging life was their only option, which may be due to receiving incomplete medical information [35]. Lack of information was identified as a significant negative factor in the transplant process during all stages for family caregivers [29].

\section{Family Caregiver Mental Health}

Effects on mental health in family caregivers in this population have been noted in multiple studies [17, 18, 27, 34]. Increased depression and anxiety have been reported in caregivers of those with HE [17, 27]. Family caregivers suffered from anxiety and depression during the waiting process for liver transplantation [19,22]. Worse psychological symptoms were seen in family caregivers who experienced a decrease in income, with Black participants more likely to experience decreases in pay related to unemployment from caregiving responsibilities [17]. Family caregivers of patients with alcohol-associated liver disease showed more depression and burden compared with family caregivers of patients with liver disease due to other etiologies [34]. One study demonstrated that patient illness severity and functional needs were associated with increased levels of depression and increased caregiver burden [24].
Increased rates of depression, low mental QOL, and mood disturbance were associated with higher levels of burden experienced by family caregivers of patients after liver transplantation [37, 38]. In a study of depressive symptoms among solid organ transplant recipients, family caregivers of people with liver transplants had increased prevalence of depression compared with caregivers of kidney transplant recipients [37]. Higher levels of depression were associated with longer time after transplant, higher perceived caregiving overload, and a lower sense of personal gain [16]. Mental health of family caregivers can also be affected by the mental health of the patients. Worse patient mental health in patients after transplant was associated with increased depression and anxiety in family caregivers [39].

\section{Coping Style and Social Support}

Few studies have examined strategies used by caregivers of patients with advanced liver disease to cope and manage caregiving. One study examined relationships among burden, coping styles, and psychological adjustment among caregivers of potential candidates for lung, liver, heart, or kidney transplantation [24]. Coping strategies included resignation, avoidance, information seeking, and social support. Only negative coping strategies were associated with depression. Greater caregiver burden and the use of avoidance were associated with higher levels of depression and anxiety. Liver transplant candidates and recipients, and their family caregivers, reported prioritizing self-management tasks related to medical and role-related tasks over tasks related to emotional management [40]. A higher level of depression was associated with greater caregiver burden and the use of resignation as a coping strategy [24]. When family caregivers in one study were asked about what coping strategies they found most helpful, they reported focusing on the needs of the patients and not themselves. Another way of coping described by caregivers was to seek information about the patient's disease as a form of empowerment [41]. In a study to investigate how perceived social support to family caregivers was associated with symptoms experienced by patients awaiting liver transplantation, social support did not predict their symptoms. However, in patients diagnosed with alcohol-related diseases, their narrower social network was associated with an increase in caregiver strain [42.••].

Little is known about positive aspects of caregiving and how they might improve family caregiver experience of those caring for people with advanced liver disease. The most common benefits include helping and spending time with the patient. Caregiver social functioning was found to be the only significant predictor of caregiver distress, with those caregivers who report greater distress also reporting interference with normal social activities [18]. Family caregivers of decedent patients who were never able to receive 
a liver transplant had some positive feelings about having done everything in their power for the patient; however, this was tempered with feelings of regret [23].

\section{Interventions}

Studies of prospective interventions targeting family caregivers of people with advanced liver disease are sparse. One recent study compared the interventions of liver disease education and self-management in patient-family caregiver dyads awaiting liver transplantation, but did not find any significant improvement in patient or caregiver outcomes; these included illness uncertainty (Perception of Uncertainty Scale-Family Member), uncertainty management (the SelfControl Schedule), self-efficacy (self-efficacy scale), and caregiver reaction (Caregiver Reaction Assessment) [11]. Another study of a 4-week mindfulness-based stress reduction and group therapy intervention showed reductions in family caregiver burden (Zarit Burden Interview), perceived burden (Perceived Caregiver Burden), and reported levels of depression (Beck Depression Inventory) among family caregivers [12].

Few palliative care interventions have been described. A nurse-led palliative care intervention was feasible, acceptable, and led to a reduction in symptom burden (Palliative Care Outcome Scale) and improved QOL (Caregiver Quality of Life Questionnaire) for family caregivers [13.••]. Patients and family caregivers have reported limited understanding of palliative care, and associated palliative care with the end

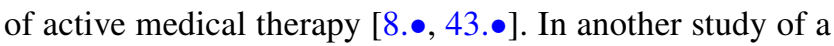
palliative care intervention in the ICU setting, family satisfaction was higher with overall care, symptom management, and end-of-life management for patients receiving the intervention [44]. We found no large scale, multi-site trials that focused on caregiver or dyadic outcomes for palliative care interventions; however, Verma and colleagues published their study protocol of the PAL-Liver study, which includes dyadic enrollment and family-caregiver-focused secondary outcomes [14].

\section{Discussion}

Family caregivers of people with advanced liver disease face significant caregiver burden that has the potential to adversely affect their QOL and their physical and mental health. There are certain sub-sets of the patient and family caregiver population facing advanced liver disease who are at higher risk of burden and lower QOL. Study populations can be stratified for risk factors of increased family caregiver burden based on the presence of HE and severity of liver disease, [27] etiology of liver disease, [34] severity of symptoms, [42.••] race, [17] gender, [16, 38] site of care (e.g.,
ICU), [45] timing after receiving a liver transplant, [39] timing relative to patient death, [46] and perceived quality of

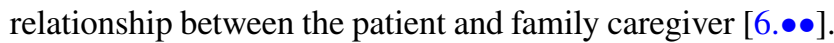
A study by Hansen and colleagues is ongoing and includes additional focus on the impact of symptom burden on patient and family caregiver dyads [47].

We identified no prior studies that systematically reviewed the literature about informal caregiving in the context of advanced liver disease and palliative care. Overall our search and subsequent article review yielded relatively few articles that discuss the experience, nature of burden, and factors that affect QOL of family caregivers of people with advanced liver diseases. About a third of the studies we found were qualitative; the high proportion of qualitative methods and descriptive studies may signify the embryonic state of the science in this area. Despite the paucity of studies, we found, a wide variety of survey tools had used, making comparison of outcomes across studies challenging.

We reviewed the results of our search and used them to create a conceptual model of family caregiving in the context of advanced liver diseases (Figure 2). We included socioeconomic factors (figure 2, upper-most box) to acknowledge the studies we found that found differential rates of burden based on gender, age, race, location, and employment status. These factors in turn affect the health status of family caregivers and patients, and the availability and quality of supports available to the family caregiver, like home services, knowledge and illness understanding, communication with medical providers, and community-based supports. We did not identify studies that cite insurance status as a sociodemographic variable impacting burden.

Family caregiver-based factors (figure 2, left middle box) that may impact burden include psychological symptoms, medical co-morbidities, coping strategies, spirituality, and the nature of the relationship with the patient (e.g., spouse versus adult child for instance and the quality of the relationship). We also identified patient-based factors (figure 2 , right middle box) that the studies in our review support as impacting family caregiver experience. These include severity of liver disease and symptoms, HE, liver transplant candidacy and wait-list status, concomitant alcohol use, and the prognosis of the patient and in particular prognostic uncertainty. Uncertainty featured prominently in our results as adversely affecting family caregivers as they care for people with liver disease.

A number of factors that impact family caregiving experience in the setting of other serious illnesses did not come up in our systematic review (figure 2, shown in yellow text). These include co-morbidities of the family caregiver, the role of spirituality, availability of home health services, community supports (e.g., church, caregiver support groups), and existential forms of burden. While location relative to primary treatment site was a theme in our 
Fig. 2 A conceptual model of family caregiving for people with advanced liver disease, focusing on factors that impact family caregiver QOL, developed based on the findings of the systematic review. Elements shown in italicized yellow text were not reflected in the results of the systematic review

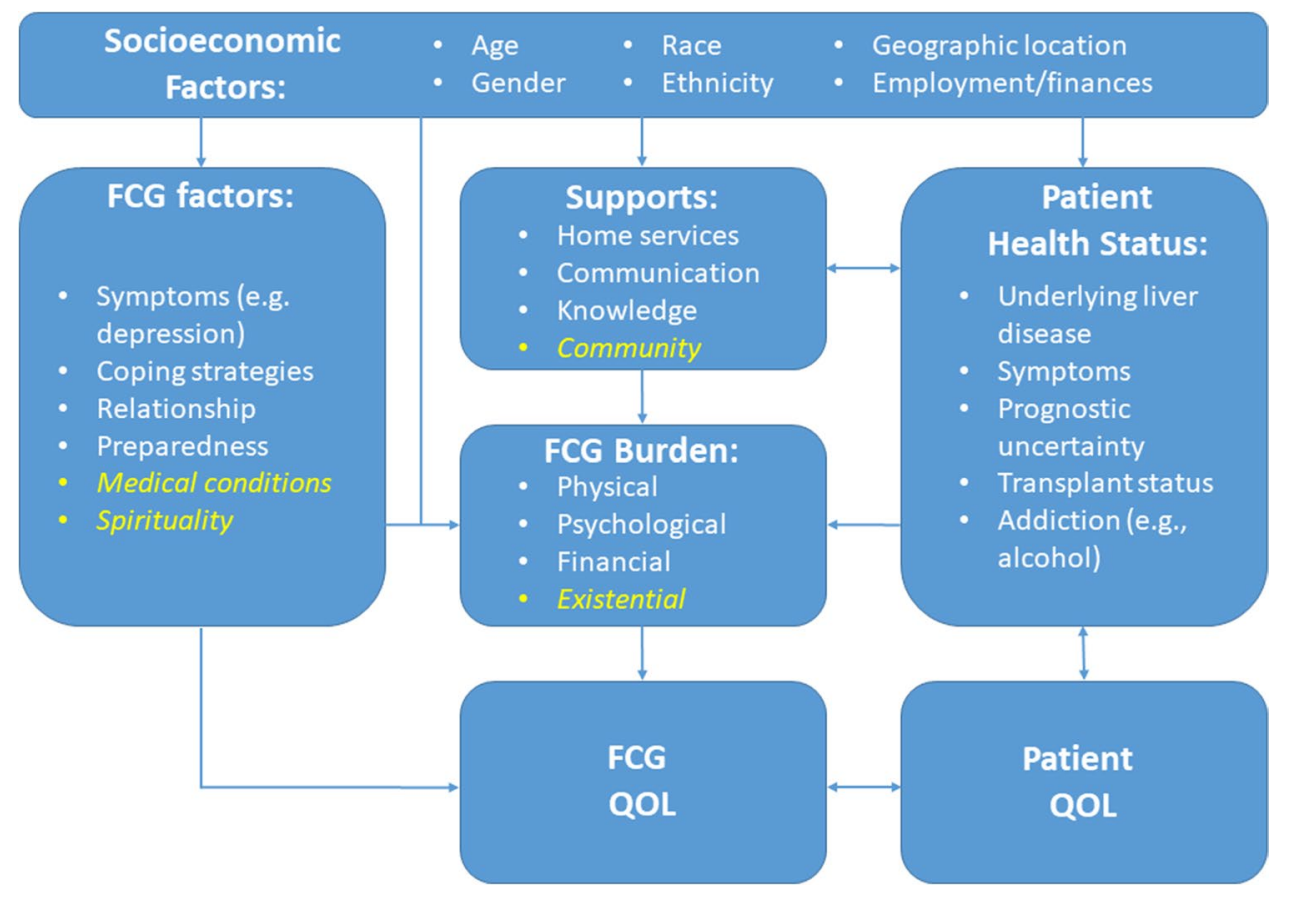

results, only two studies from Taiwan focused on receipt of care in mainland China $[15,30]$. We did not find studies of the experience of family caregivers of patients who need to travel from rural areas in the USA for specialized liver care. We also found little about availability and compatibility of medical and support resources available for care at home to augment family caregiver efforts for people with advanced liver disease [21.•].

The themes addressed overall by the studies we found suggest that family caregiver QOL is amenable to palliative care interventions. Given the intersection of factors including social, physical, psychological, complex care systems, uncertainty, and potentially existential or spiritual factors, the multi-faceted approach of palliative care is well-suited to address many of the unmet needs of this population [7].

Additional observational and cross-sectional studies will benefit this field. A better understanding is needed of the changing types and magnitude of family caregiver needs across the trajectory advanced liver disease. More information is needed about which sub-populations would most benefit from interventions. For example, given rising rates of non-alcoholic steatohepatitis (NASH), HCC, and alcohol-associated liver disease in younger patients, what are the different physical and psychosocial needs at various milestones of these disease processes [3, 48]? How will changing organ allocation policies affect the experience of family caregivers of people with different types of liver diseases? What are the positive aspects of caregiving that could serve as a foundation for future interventions? Future efforts to develop QOL interventions for family caregivers-palliative care or otherwise-should address these questions to best understand the timing of the intervention and optimal inclusion criteria, and incorporate the interdependent nature of the patient and family caregiver experiences $[6 . \bullet \bullet]$.

Our study has important strengths. First, this is a novel systematic review in an area that has seen no similar prior publications. Second, we searched multiple databases with the help of a health sciences librarian co-investigator to ensure completeness of our results. Finally, the search results were reviewed by multiple investigators with blinding of others' assessments to reduce bias and ensure consistency.

This study also has limitations. First, it was not feasible to include studies written in languages other than English, and a few studies were excluded on this basis. Second, our search did not include systematic review of family caregiving in other illness populations, so our comparisons outside of the context of liver diseases relied on the expertise of the authors of this review. Finally, we have not included an assessment of bias in our inclusion criteria given the very few studies in this area. Future work should focus on evaluation of the quality of study design and selection of outcomes, as more work is published in this area of growing interest.

\section{Conclusion}

Our study brings to light the significant physical, psychological, and financial burden of family caregivers of patients with advanced liver disease that have been reported in the 
scientific literature. The studies we found report factors leading to increased burden and decreased QOL of family caregivers including poor mental health, unpredictability of clinical course/trajectory, presence of HE, and lack of information and communication. There is a scarcity of literature describing the nature of burden among family caregivers of people with advanced liver disease or successful strategies for mitigating this burden. Our review identifies few family caregiverfocused interventions in this population, including those in palliative care. We describe a model based on our findings that we believe could aid in conceptualization of future studies to comprehensively address all types of burden in this population.

Supplementary Information The online version contains supplementary material available at https://doi.org/10.1007/s11901-021-00575-9.

Acknowledgments The work reported on in this publication is supported in part by the National Institute of Nursing Research (NINR) of the National Institutes of Health (NIH) under award number R01 NR016017 (LH) and the National Institute on Aging (NIA) of the NIH under award number R03 AG067992 (CW, LH). The views expressed in this article are those of the authors and do not necessarily reflect the positions or policies of the NIH, the Department of Veterans Affairs, or the US Government.

\section{Declarations}

Conflict of Interest Christopher Woodrell, Arnab Mitra, Andrew Hamilton, and Lissi Hansen declare that they have no conflict of interest.

Human and Animal Rights and Informed Consent This article does not contain any studies with human or animal subjects performed by any of the authors.

Open Access This article is licensed under a Creative Commons Attribution 4.0 International License, which permits use, sharing, adaptation, distribution and reproduction in any medium or format, as long as you give appropriate credit to the original author(s) and the source, provide a link to the Creative Commons licence, and indicate if changes were made. The images or other third party material in this article are included in the article's Creative Commons licence, unless indicated otherwise in a credit line to the material. If material is not included in the article's Creative Commons licence and your intended use is not permitted by statutory regulation or exceeds the permitted use, you will need to obtain permission directly from the copyright holder. To view a copy of this licence, visit http://creativecommons.org/licenses/by/4.0/.

\section{References}

Papers of particular interest, published recently, have been highlighted as:

- Of importance

$\bullet$ Of major importance

1. Centers for Disease Control and Prevention: National Center for Health Statistics. Cdc.gov/nchs/fastats/liver-disease.htm. Accessed October 2021
2. Gomez EV, Rodriguez YS, Bertot LC, et al. The natural history of compensated HCV-related cirrhosis: a prospective long-term study. J Hepatol. 2013;58(3):434-44.

3. Kwong A, Kim WR, Lake JR, et al. OPTN/SRTR 2018 annual data report: liver. Am J Transplant. 2020;20(Suppl s1):193-299.

4. U.S. Department of Health and Human Services aUSDoL. The future supply of long-term care workers in relation to the aging baby boom generation: report to Congress. Washington, DC: Office of the Assistant Secretary for Planning and Evaluation2003.

5. Rabiee A, Ximenes RO, Nikayin S, Hickner A, Juthani P, Rosen RH, Garcia-Tsao G. Factors associated with health-related quality of life in patients with cirrhosis: a systematic review. Liver Int. 2021;41(1):6-15.

6.•• Hansen L, Chang M, Lee C, Hiatt S, Dieckmann NF, Lyons K. Physical and mental quality of life in patients with end-stage liver disease and their informal caregivers. Clin Gasterenterol Hepatol. 2020(in press). https://doi.org/10.1016/j.cgh.2020.04. 014 . This dyadic $(n=132$ dyads) cross-sectional study identified characteristics of patients with end-stage liver disease and their family caregivers that were associated with quality of life. Findings showed: (1) high levels of uncertainty were associated with worse physical and mental quality of life for patients and caregivers; (2) refractory ascites was associated with worse physical quality of life for both of them; (3) a history of hepatic encephalopathy was associated with worse physical quality of life for patients; and (4) relationship quality was associated with mental quality of life for patients. Dyadic research is needed to gain knowledge on how to optimize health outcomes for both members of the patient-caregiver dyad.

7. Kelley AS, Morrison RS. Palliative care for the seriously ill. N Engl J Med. 2015;373(8):747-55.

8. Low JTS, Rohde G, Pittordou K, Candy B, Davis S, Marshall A, et al. Supportive and palliative care in people with cirrhosis: International systematic review of the perspective of patients, family members and health professionals. J Hepatol 2018;69(6):1260-73. https://doi.org/10.1016/j.jhep.2018.08. 028 . This systematic review employed three major biomedical databases to identify articles about patient, family, and healthcare professional perspectives on the provision of care in cirrhosis, which were rigorously appraised by the investigators. Nineteen studies were identified, showing that patients and families lacked information and understanding of cirrhosis, healthcare professionals struggled communicating with patients and families, and general practitioners lacked confidence to discuss prognosis and care preferences with patients and families. Palliative care was cited as needing to be integrated earlier into community and specialty practices for this population.

9. Shamseer L, Moher D, Clarke M, et al. Preferred reporting items for systematic review and meta-analysis protocols (PRISMA-P) 2015: elaboration and explanation. BMJ. 2015;350:g7647.

10. Ouzzani M, Hammady H, Fedorowicz Z, Elmagarmid A. Rayyan-a web and mobile app for systematic reviews. Syst Rev. 2016;5(1):210.

11. Bailey DE Jr, Hendrix CC, Steinhauser KE, et al. Randomized trial of an uncertainty self-management telephone intervention for patients awaiting liver transplant. Patient Educ Couns. 2017;100(3):509-17.

12. Bajaj JS, Ellwood M, Ainger T, et al. Mindfulness-based stress reduction therapy improves patient and caregiver-reported outcomes in cirrhosis. Clin Transl Gastroenterol. 2017;8(7):e108.

13.• Kimbell B, Murray SA, Byrne H, Baird A, Hayes PC, MacGilchrist A, et al. Palliative care for people with advanced liver disease: A feasibility trial of a supportive care liver nurse specialist. 
Palliat Med. 2018;32(5):919-29. https://doi.org/10.1177/02692 16318760441. This prospective, single-arm pilot study $(\mathbf{n}=$ 47 patients and $n=27$ family caregivers) examined feasibility and acceptability of a six-month longitudinal nurse-led palliative and supportive care intervention. The intervention was feasible, acceptable, and the recruitment and outcome measurement procedures will be refined and incorporated into a future randomized controlled trial.

14. Verma M, Kosinski AS, Volk ML, et al. Introducing palliative care within the treatment of end-stage liver disease: the study protocol of a cluster randomized controlled trial. J Palliat Med. 2019;22(S1):34-43.

15. Chen HM, Shih FJ, Chang CL, Lai IH, Shih FJ, Hu RH. Caring for overseas liver transplant recipients: Taiwan primary family caregivers' experiences in mainland China. Transplant Proc. 2010;42(10):3921-3.

16. Cohen M, Katz D, Baruch Y. Stress among the family caregivers of liver transplant recipients. Prog Transplant. 2007;17(1):48-53.

17. Bolden L, Wicks MN. Predictors of mental health, subjective burden, and rewards in family caregivers of patients with chronic liver disease. Arch Psychiatr Nurs. 2010;24(2):89-103.

18. Meltzer LJ, Rodrigue JR. Psychological distress in caregivers of liver and lung transplant candidates. J Clin Psychol Med Settings. 2001;8(3):173-80.

19. Malik P, Kohl C, Holzner B, et al. Distress in primary caregivers and patients listed for liver transplantation. Psychiatry Res. 2014;215(1):159-62.

20. Hansen L, Rosenkranz SJ, Wherity K, Sasaki A. Living with hepatocellular carcinoma near the end of life: family caregivers' perspectives. Oncol Nurs Forum. 2017;44(5):562-70.

21. Hudson B, Hunt V, Waylen A, McCune CA, Verne J, Forbes $\mathrm{K}$. The incompatibility of healthcare services and end-of-life needs in advanced liver disease: A qualitative interview study of patients and bereaved carers. Palliat Med. 2018;32(5):908-18. https://doi.org/10.1177/0269216318756222. This cross-sectional qualitative study explored end-of-life needs of people with liver disease $(n=12)$ and their family caregivers ( $=5$ ), how well existing services meet their needs, and attitudes toward palliative care. The study found that available resources to the study population do not match the needs that exist near the end of life.

22. Dominguez-Cabello E, Perez-San-Gregorio MA, Martin-Rodriguez A, Perez-Bernal J. Comparison of anxious and depressive symptomatology among pretransplant hepatic patients and their relatives. Transplant Proc. 2010;42(8):2962-3.

23. Heyink J, Tymstra T. Liver transplantation: the shadow side. Fam Pract. 1990;7(3):233-7.

24. Goetzinger AM, Blumenthal JA, O'Hayer CV, et al. Stress and coping in caregivers of patients awaiting solid organ transplantation. Clin Transplant. 2012;26(1):97-104.

25. Shih W-MJ, Hsiao P-J, Chen M-L, Lin M-H. Experiences of family of patient with newly diagnosed advanced terminal stage hepatocellular cancer. Asian Pac J Cancer Prev. 2013;14(8):4655-60.

26 Roth K, Lynn J, Zhong Z, Borum M, Dawson NV. Dying with end stage liver disease with cirrhosis: insights from SUPPORT. Study to Understand Prognoses and Preferences for Outcomes and Risks of Treatment. J Am Geriatr Soc. 2000;48(5 Suppl):S122-130.

27. Bajaj JS, Wade JB, Gibson DP, et al. The multi-dimensional burden of chirrosis and hepatic encephalopathy on patients and caregivers. Am J Gastroenterol. 2011;106(9):1646-53.

28. Rakoski MO, McCammon RJ, Piette JD, Iwashyna TJ. Burden of cirrhosis on older Americans and their families: analysis of the health and retirement study. Hepatology. 2012;55(1):184-91.
29. Chen H-M, Hu R-H, Shih F-J, Shih F-J. Dilemmas across different overseas liver transplant stages: Taiwan transplant recipient families' perspectives. Transplant Proc. 2012;44(2):539-43.

30. Chen HM, Jong Shih F, Chang CL, Lai IH, Shih FJ, Hu RH. Caring for overseas liver transplant recipients: Taiwan primary family caregivers' experiences in mainland China. Transplant Proc. 2010;42(10):3921-3.

31. Kunzler-Heule P, Beckmann S, Mahrer-Imhof R, Semela D, Handler-Schuster D. Being an informal caregiver for a relative with liver cirrhosis and overt hepatic encephalopathy: a phenomenological study. J Clin Nurs. 2016;25(17-18):2559-68.

32. Kimbell B, Boyd K, Kendall M, Iredale J, Murray SA. Managing uncertainty in advanced liver disease: a qualitative, multiperspective, serial interview study. BMJ Open. 2015;5(11):e009241-e009241.

33. Montagnese S, Amato E, Schiff S, et al. A patients' and caregivers' perspective on hepatic encephalopathy. Metab Brain Dis. 2012;27(4):567-72.

34. Miyazaki ET, dos Santos RJ, Miyazaki MC, et al. Patients on the waiting list for liver transplantation: caregiver burden and stress. Liver Transplant. 2010;16(10):1164-8.

35. Hansen L, Press N, Rosenkranz SJ, et al. Life-sustaining treatment decisions in the ICU for patients with ESLD: a prospective investigation. Res Nurs Health. 2012;35(5):518-32.

36. Hansen L, Yan Y, Rosenkranz SJ. The power of the liver transplant waiting list: a case presentation. Am J Crit Care. 2014;23(6):510-5.

37. Bolkhir A, Loiselle MM, Evon DM, Hayashi PH. Depression in primary caregivers of patients listed for liver or kidney transplantation. Prog Transplant. 2007;17(3):193-8.

38. Rodrigue JR, Dimitri N, Reed A, Antonellis T, Hanto DW, Curry M. Quality of life and psychosocial functioning of spouse/partner caregives before and after liver transplantation. Clin Transplant. 2011;25(2):239-47.

39. Pérez-San-Gregorio MA, Martín-Rodríguez A, Pérez-Bernal J. Influence of the psychological state of relatives on the quality of life of patients at 1 year after transplantation. Transplant Proc. 2008;40(9):3109-11.

40. Beckmann S, Kunzler-Heule P, Biotti B, Spirig R. Mastering together the highs and lows: patients' and caregivers' perceptions of self-management in the course of liver transplantation. Prog Transplant. 2016;26(3):215-23.

41. Lasker JN, Sogolow ED, Sharim RR. For better and for worse: family and friend's responses to chronic liver disease. Illness Crisis Loss. 2005;13(3):249-66.

42.•• Cipolletta S, Entilli L, Nucci M, Feltrin A, Germani G, Cillo U, et al. Psychosocial support in liver transplantation: A dyadic study with patients and their family caregivers Frontiers in Psychology. 2019;10(2304). https://doi.org/10.3389/fpsyg.2019. 02304 . This cross-sectional quantitative study examined perceived social support and dependency and their association with the psychological wellbeing of liver transplant patients $(n=51)$ and their family caregivers $(n=44)$. Perceived social support and dependency did not predict patient and caregiver symptoms and family strain was significantly associated with worsening of caregiver and patient wellbeing. These findings support the importance of evaluating both members of the dyad in liver transplantation.

43.• Donlan J, Ufere NN, Indriolo T, Jackson V, Chung RT, ElJawahri A, et al. Patient and Caregiver Perspectives on Palliative Care in End-Stage Liver Disease. J Palliat Med. 2020. https:// doi.org/10.1089/jpm.2020.0551. This cross-sectional qualitative study explored knowledge, perceptions, and preferences about palliative care of transplant-listed and transplant-ineligible patients with end-stage liver disease $(n=15)$ and their 
informal caregivers $(n=14)$. Participants had no or limited knowledge of palliative care and frequently associated it end-of-life care. Once provided a definition of palliative care, most participants thought that palliative care should be integrated early on into end-stage liver disease management.

44. Lamba S, Murphy P, McVicker S, Harris Smith J, Mosenthal AC. Changing end-of-life care practice for liver transplant service patients: structured palliative care intervention in the surgical intensive care unit. J Pain Symptom Manage. 2012;44(4):508-19.

45. Perez-San-Gregorio MA, Martin-Rodriguez A, Asian-Chavez E, Gallego-Corpa A, Perez-Bernal J. Psychological adaptation of liver transplant recipients. Transplant Proc. 2005;37(3):1502-4.

46. Roth K, Lynn J, Zhong Z, Borum M, Dawson NV. Dying with end stage liver disease with cirrhosis: insight from SUPPORT. J Am Geriatr Soc. 2000;48(5):S122-30.

47. Hansen L, Lyons KS, Dieckmann NF, et al. Background and design of the symptom burden in end-stage liver disease patient-caregiver dyad study. Res Nurs Health. 2017;40(5):398-413.

48. Tapper EB, Parikh ND. Mortality due to cirrhosis and liver cancer in the United States, 1999-2016: observational study. BMJ. 2018;362:k2817.

49. Pérez San Gregorio MA, Martín Rodríguez A, Pérez Bernal J. Psychological differences of patients and relatives according to post-transplantation anxiety. Span J Psychol. 2008; 11(1):250-258

50. Bajaj JS, Thacker LR, Wade JB, et al. PROMIS computerized adaptive tests are dynamic instruments to measure health-related quality of life in patients with cirrhosis. Aliment Pharmacol Ther 2011; 34(9): 1123-1132

Publisher's Note Springer Nature remains neutral with regard to jurisdictional claims in published maps and institutional affiliations. 